Recepción: 05/02/2018

Aceptación: 11/03/2019

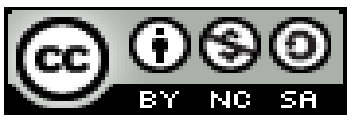

Ciencias sociales y políticas

Publicación: 05/04/2019

Artículo de investigación

\title{
Prevención de la drogadicción en niños, niñas y adolescentes de la comuna "Los Bajos del Pechiche"
}

\section{Prevention of drug addiction in children and adolescents of the commune "The Pechiche Basses"}

\section{Prevenção da toxicodependência em crianças e adolescentes da comuna "O Pechiche Basses"}

Jazmin Estefanía Moreira-Valencia ${ }^{\mathrm{I}}$

jazmin.moreira@uleam.edu.ec

Nancy Lorena Reyes-Mero II nancy.reyes@uleam.edu.ec

Patricia Josefa López-Mero ${ }^{\text {III }}$

patrícia.lopez@uleam.edu.ec

Richard Fabricio Rodríguez-Andrade IV richard.rodriguez@uleam.edu.ec

Correspondencia: nancy.reyes@uleam.edu.ec

I Magíster en Dirección de Talento Humano, Licenciada en Trabajo Social, Psicóloga Clínica, Docente de la Facultad de Trabajo Social de la Universidad Laica Eloy Alfaro de Manabí, Manta, Ecuador.

II Magíster en Trabajo Social, Licenciada en Trabajo Social, Trabajadora Social, docente de la facultad de Trabajo Social, Universidad Laica Eloy Alfaro de Manabí, Manta, Ecuador.

III Licenciada en Ciencias de la Educación: especialidad Castellano y Literatura. Licenciada en Trabajo Social, especialidad: Atención a la Familia y al Desarrollo Comunitario. Diploma Superior en Intervención Social. Magister en Gerencia Educativa. Decana de la Facultad de Trabajo Social de la Universidad Laica Eloy Alfaro de Manabí, Manta, Ecuador.

IV Magíster en Gerencia Educativa, Licenciado en Ciencias de la Educación, Analista de Sistemas, Docente de la Facultad de Trabajo Social de la Universidad Laica Eloy Alfaro de Manabí, Manta, Ecuador. 


\section{Resumen}

El estudio evalúa de forma cuantitativa, con un enfoque descriptivo el nivel de impacto obtenido después de la ejecución de un programa de prevención sobre la drogadicción en niños, niñas y adolescentes de la comuna "Los bajos del Pechiche" del cantón Montecristi, provincia de Manabí, para establecer si las acciones emprendidas generaron cambios en los niños, niñas y adolescentes de la referida población y evitar el consumo de drogas, reduciendo un problema que afecta a buena parte de la población en general; se recogió el criterio de estudiantes, docentes, padres de familia de la Unidad Educativa Fiscal "Daniel Acosta Rosales" y comunidad en general. De acuerdo con la información recopilada durante la ejecución de una encuesta y entrevistas a informantes claves, se encontraron los siguientes resultados: Los compromisos generados estuvieron en un rango medio, la participación de la comunidad fue regular, las actividades desarrolladas para el buen uso del tiempo libre fueron muy relevantes, el consumo de estupefacientes psicotrópicos si ha tenido una disminución del 51\% al 75\% dentro de la Comunidad "Los Bajos Del Pechiche” y la Unidad Educativa Dr. Daniel Acosta Rosales, se abarcó un considerable número de beneficiarios, incluyéndose a estudiantes, docentes, padres de familia y comunidad causando un impacto positivo que según los involucrados debe continuar para beneficio de los niños, niñas y adolescentes de la comunidad.

Palabras Clave: Prevención; drogadicción; padres; docentes; estudiantes. 


\begin{abstract}
The study quantitatively assesses, with a descriptive approach, the level of impact obtained after the execution of a prevention program on drug addiction in children and adolescents of the community "Los bajo del Pechiche" in the canton Montecristi, province of Manabí, to establish if the actions undertaken generated changes in the children and adolescents of the referred population and avoid drug use, reducing a problem that affects a good part of the population in general; The criteria of students, teachers, parents of the Fiscal Education Unit "Daniel Acosta Rosales" and the community in general were collected. According to the information collected during the execution of a survey and interviews with key informants, the following results were found : The commitments generated were in a medium range, the participation of the community was regular, the activities developed for the good use of free time were very relevant, the consumption of psychotropic drugs if it has had a decrease of $51 \%$ to $75 \%$ within The "Los Bajos Del Pechiche" Community and the Dr. Daniel Acosta Rosales Educational Unit, covered a considerable number of beneficiaries, including students, teachers, parents and community causing a positive impact that according to those involved should continue for the benefit of the children and adolescents of the community.
\end{abstract}

Keyword: Prevention; drug addiction; parents; teachers; students. 


\section{Resumo}

O estudo avalia quantitativamente, com abordagem descritiva, o nível de impacto obtido após a execução de um programa de prevenção à dependência de drogas em crianças e adolescentes da comunidade "Los bajo del Pechiche" do cantão de Montecristi, província de Manabí. , estabelecer se as ações realizadas geraram mudanças nas crianças e adolescentes da população referida e evitar o uso de drogas, reduzindo um problema que afeta boa parte da população em geral; Foram coletados os critérios dos alunos, professores, pais da Unidade de Educação Fiscal "Daniel Acosta Rosales" e da comunidade em geral, segundo as informações coletadas durante a realização de uma pesquisa e entrevistas com informantes-chave, foram encontrados os seguintes resultados : Os compromissos gerados foram de médio alcance, a participação da comunidade foi regular, as atividades desenvolvidas para o bom aproveitamento do tempo livre foram muito relevantes, o consumo de psicotrópicos se houve uma queda de $51 \%$ para $75 \%$ dentro $\mathrm{A}$ Comunidade "Los Bajos Del Pechiche" e a Unidade Educacional Dr. Daniel Acosta Rosales, cobriram um número considerável de beneficiários, incluindo estudantes, professores, pais e comunidade, causando um impacto positivo que, de acordo com os envolvidos, deve continuar em benefício da comunidade. as crianças e adolescentes da comunidade.

Palabras Clave: Prevenção; toxicodependência; pais; professores; alunos. 


\section{Introducción}

Para combatir el flagelo de la drogadicción lo más conveniente es la prevención, dado que los casos de jóvenes que padecen esta condición, son casos difíciles para su regeneración y más aún, para su curación. La drogadicción se ha convertido en un problema mundial de larga data que debe analizarse a nivel estudiantil; y los maestros deben abordar el problema desde un enfoque preventivo, por tanto, deben apertrecharse de conocimientos e información pertinente y relativa al tema: su historia, las características de las adicciones, clases de adicción, dado que, para realizar actividades preventivas, el tema les compete.

Asimismo, es relevante que los docentes sepan el daño que el uso de narcóticos, alucinantes, estimulantes, alcohol o tabaco podrían causarle al organismo humano, por lo que, el control sobre los psicotrópicos en general, es fundamental. Aunado a ello, la familia y la escuela conforman el pilar fundamental que otorga formación de valores en los niños y jóvenes, por ello es necesario atender y coadyuvar hacia la solución de problemas de comunicación, de recursos económicos, de relaciones interpersonales, de falta de conocimiento, de atención individualizada de la matrícula, entre otros.

La drogodependencia es un fenómeno antiquísimo, se conocen referencias desde el año 2737 A.C., en China, en Grecia, en Roma, en la cultura Azteca, entre otros. Desde este marco, el incremento de la toxicomanía ha evolucionado con el paso del tiempo y refleja la tensión de situaciones sociales, tales como maltratos, barreras comunicativas, precariedad de recursos económicos, entre otros. Para combatir situaciones adversas que puedan ser asumidas como condiciones proclives hacia la drogodependencia, como amenaza para la salud integral de los individuos, la escuela ejerce su papel junto a la comunidad y a la familia. Por tanto, es necesario conocer las opiniones que poseen los estudiantes y docentes acerca de este tema, específicamente desde un enfoque preventivo.

Es así como, en la Unidad Educativa "Daniel Acosta Rosales," adscrita a la comuna "Los Bajos del Pechiche" se ejecutó un programa de prevención de drogas con alcance comunitario, que incluyó charlas preventivas, integrándose actividades deportivas y recreativas para el correcto uso del tiempo libre. Al término del mencionado programa se aplicaron encuesta y entrevistas para verificar el nivel de impacto obtenido, con resultados satisfactorios. 


\section{Materiales y Métodos}

\section{Fundamentos básicos referenciales sobre la drogadicción}

En este apartado se abordan los fundamentos básicos sobre la droga, la drogadicción y la prevención. En ese sentido se coincide con García Hoz (2000: p. 110), quien aporta que la droga es "una sustancia... de la que se abusa por auto-administración para fines distintos de los legítimos en medicina, y que pueden introducir una modificación en el organismo humano cambiando las ideas o sentimientos del hombre" de lo que se deduce que la droga puede modificar el comportamiento cotidiano de quien se la administra.

Estos cambios pueden resultar placenteros, pueden generar dependencia y la necesidad de administrarse cada vez más cantidad de la sustancia, creando en su organismo una dependencia que incide en la salud física y psicológica. Dentro de estas sustancias se encuentran los sedantes, los barbitúricos, el alcohol, tranquilizantes, estimulantes y alucinógenos, entre otros.

Expertos como García Hoz (Op cit) consideran que la drogadicción ocurre en diversos estratos sociales pero diversos estudios a lo largo del tiempo coinciden en que la clase social de bajos recursos es vulnerable por diversas condiciones socioeconómicas adversas. Asimismo, considera el precitado autor que, la drogadicción puede deberse a varios factores entre los que destacan familias conflictivas, disfuncionales, que no apoyan a los hijos, sin formación religiosa, con problemas de valores como la disciplina y otros factores.

Lo anterior puede suscitar insatisfacción en niños y adolescentes, llevándolos a sentir curiosidad por sensaciones desconocidas, sentir inadaptación psicológica y predisposición al uso de sustancias que impliquen "un escape" de la realidad vivida, a sentir una situación emocional difícil y depresiva hasta llegar a la vulnerabilidad por falencia de una sólida formación en valores, en moral y ética por parte de la familia y la institución educativa.

Es ante este panorama posible y amenazante de la sociedad, la familia, la escuela, la educación, el contexto comunitario y los docentes cobran un papel relevante en cuanto a la formación de valores se refiere, como fundamento básico de abordaje del tema de la drogadicción, desde el enfoque de la prevención, desde los procesos de orientación y formación ética, donde la familia ejerza su rol de apoyo, de soporte, de control, de afectividad, comprensión, orientación y la 
escuela en forma mancomunada consolide la formación de la personalidad con acciones positivas que generen conciencia y desarrollen personas sanas.

Los resultados del nivel de impacto y relevancia del programa de prevención de drogas fueron obtenidos con la aplicación de encuestas a 342 miembros de la comunidad Los Bajos del Pechiche, 333 estudiantes, 73 padres de familia, 17 docentes y 7 profesionales entre directivos y miembros del DECE de la Unidad Educativa "Daniel Acosta Rosales" y 10 entrevistas dirigidas a la rectora de la institución, analista del DECE, 4 padres de familia y 4 estudiantes, con preguntas relevantes sobre el programa de prevención aplicado, su aceptación y los cambios producidos en los beneficiarios. En ese contexto, esta investigación se orientó hacia el marco metodológico sobre la base de un estudio descriptivo. Al respecto, Arias (2006, p. 45); señala que: "La investigación descriptiva consiste en la caracterización de un hecho, fenómeno o grupo, con el fin de establecer su estructura o comportamiento". Los resultados de este tipo de investigación se ubican en un nivel intermedio en cuanto a la profundidad de los conocimientos se refiere.

En este mismo orden de ideas, Hernández, Fernández y Baptista (2006), reseñan que la investigación descriptiva, tienen como propósito la búsqueda específica de las propiedades significativas de las personas, grupos, comunidades, instituciones o cualquier otro fenómeno que sea susceptible al análisis (p. 125). Con base en estas definiciones, se aplicó este tipo de investigación al estudio actual, ya que se tomó en cuenta a los niños, niñas y adolescentes de la Comuna "Los Bajos del Pechiche", basados en la prevención de la drogadicción en dicha Comuna y el impacto producido.

En este sentido, es consideración de los autores asumir que los estudios descriptivos miden la información de manera independiente o conjunta sobre los conceptos o las variables a las que se refieren, se van describiendo sus características según los objetivos establecidos. Esta investigación se desarrolló bajo el enfoque descriptivo, ya que describe cualidades y caracteres, que muestran los integrantes de la población objeto de estudio tal cual como se vayan presentando en el sitio de los acontecimientos.

\section{Diseño de la Investigación}

En primer lugar, se declara que ésta se trata de una investigación con diseño de campo, cuya toma de datos se hizo directamente de una fuente primaria, viva, es decir los niños, niñas y 
adolescentes, padres de familia, docentes, directivo e integrantes de la Comuna "Los Bajos del Pechiche" y de la Unidad Educativa "Daniel Acosta Rosales" de la misma zona. En ese orden se siguen los enfoques de Arias (2006), para quien el diseño de campo “...consiste en la recolección de datos directamente de los sujetos investigados, o de la realidad donde ocurren los hechos (datos primarios), sin manipular o controlar variable alguna, es decir el investigador obtiene la información, pero no altera las condiciones existentes" (p.31).

En lo concerniente a la manipulación o no de la variable, es importante resaltar que este diseño se consideró como no experimental, pues su fin no consistió en observar el cambio que una o unas variables podrían sufrir al estar expuesta a la influencia de otra, sino que sólo se analizó la variable: "Prevención de la drogadicción en la Comuna "Los Bajos de Pechiche" y su nivel de impacto.

Por último, en función del número de mediciones a lo largo de un periodo de tiempo, se tiene que el diseño fue transeccional o transversal, que según Hernández, Fernández y Baptista (op. cit) son diseños en los que se "recolectan datos en un solo momento, en un tiempo único" (p.208). De este modo, los datos fueron recogidos durante una única administración del instrumento elaborado para la recolección de los datos. Aunado a ello, la Universidad Pedagógica Experimental Libertador (2006, p. 5), define la investigación de campo como:

El análisis sistemático de los problemas en la realidad con el propósito, bien sea de describirlos, interpretarlos, entender su naturaleza y factores constituyentes, explicar sus causas y efectos, o predecir su ocurrencia, haciendo uso de métodos característicos de cualquiera de los paradigmas o enfoques de investigación conocidos o en desarrollo.

En relación con este estudio, los datos requeridos para efectuar el nivel de impacto fueron recabados directamente por los autores de la presente investigación, se aplicó un instrumento tipo encuesta a los estudiantes y docentes de La Institución Educativa "Daniel Acosta Rosales", en cuanto al nivel de impacto que tuvo el programa de Prevención de la drogadicción en la institución educativa. 


\section{Fases Metodológicas de la Investigación}

Para facilitar el desarrollo de esta investigación se procedió a realizar las siguientes actividades:

Se revisó bibliografía específica y general, referida a la temática investigada, con este fin se examinaron fuentes como textos especializados, consultas electrónicas, periódicos, trabajos de investigación, revistas y fuentes legales, los cuales fueron reseñados en el estudio, de igual manera, se elaboró la conceptualización y operacionalización de las variables.

Por otro lado, se tabularon y se procesaron las respuestas obtenidas en forma manual y, las mismas fueron vaciadas en cuadros, donde se ubicaron las frecuencias y porcentajes de las respuestas obtenidas. Se procesó la información, se efectuó un análisis descriptivo-cuantitativo e interpretativo de los resultados, simultáneamente vinculando los sustentos teóricos del estudio con la información recabada. Seguidamente, al análisis se establecieron las conclusiones y recomendaciones pertinentes a que fueron lugar, acordes con los resultados del trabajo de investigación científica.

\section{Resultados y Discusión}

De la aplicación de la encuesta aplicada a todos los actores involucrados en el programa de prevención, se obtuvo una serie de resultados, tal como se presentan a continuación:

Tabla 1. Los compromisos generados por la comunidad para el desarrollo del proyecto alcanzaron

\begin{tabular}{|c|c|c|c|c|c|}
\hline Compromisos & Comunidad & Estudiantes & $\begin{array}{c}\text { Padres de } \\
\text { familia }\end{array}$ & Docentes & $\begin{array}{c}\text { Directivos } \\
\text { y DECE }\end{array}$ \\
\hline $25 \%$ & $27 \%$ & $10 \%$ & $25 \%$ & $0 \%$ & $0 \%$ \\
\hline $50 \%$ & $39 \%$ & $31 \%$ & $27 \%$ & $24 \%$ & $17 \%$ \\
\hline $75 \%$ & $24 \%$ & $36 \%$ & $48 \%$ & $76 \%$ & $83 \%$ \\
\hline $100 \%$ & $9 \%$ & $23 \%$ & $0 \%$ & $0 \%$ & $0 \%$ \\
\hline Sin conocimiento & $1 \%$ & $0 \%$ & $0 \%$ & $0 \%$ & $0 \%$ \\
\hline Total & $100 \%$ & $100 \%$ & $100 \%$ & $100 \%$ & $100 \%$ \\
\hline
\end{tabular}

Fuente: La Comunidad Los Bajos Del Pechiche y la Unidad Educativa Dr. Daniel Acosta Rosales

Realizado por: Estudiantes de Octavo semestre de la Facultad de Trabajo Social, Uleam.

Gráfico 1. Los compromisos generados por la comunidad para el desarrollo del proyecto alcanzaron 


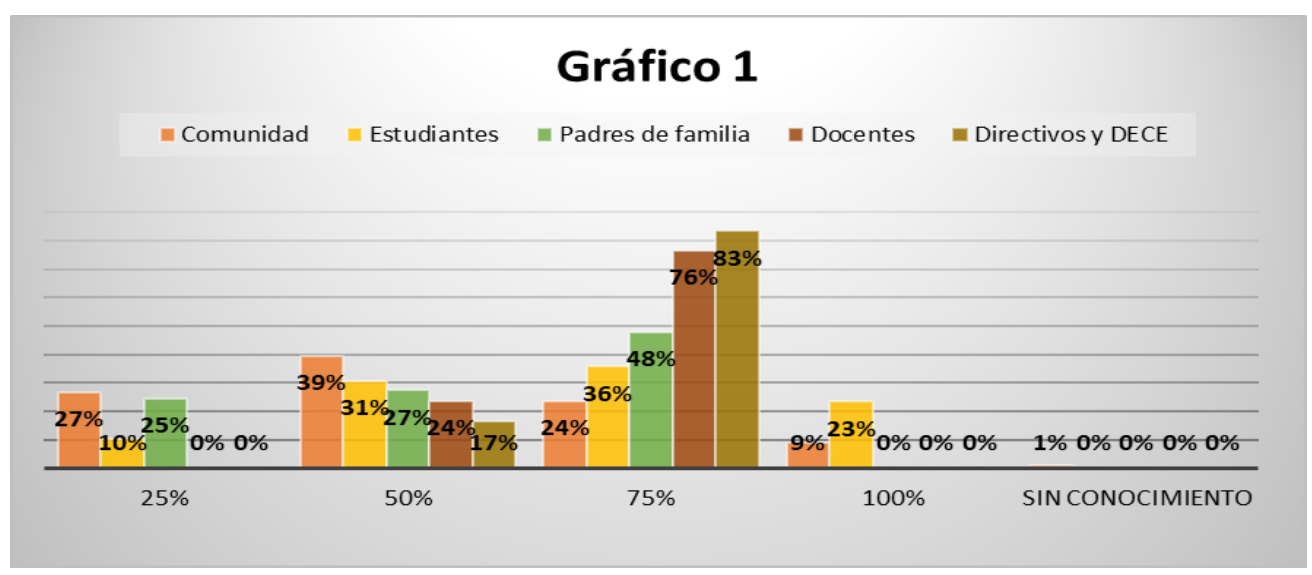

Fuente: La Comunidad Los Bajos Del Pechiche y la Unidad Educativa Dr. Daniel Acosta Rosales

Realizado por: Estudiantes de Octavo semestre de la Facultad de Trabajo Social, Uleam.

\section{Análisis:}

De acuerdo a la información obtenida por la encuesta realizada a La Comunidad "Los Bajos Del Pechiche y a la Unidad Educativa Siglo XXI Dr. Daniel Acosta Rosales indican que se los compromisos generados lo determinan la mayor parte de la muestra poblacional en un $50 \%$ de los compromisos.

Tabla 2. ¿Cómo fue la participación de los involucrados en el proyecto de prevención de drogas en los Bajos del Pechiche?

\begin{tabular}{|l|c|c|c|c|c|}
\hline $\begin{array}{l}\text { Participación de } \\
\text { involucrados }\end{array}$ & Comunidad & Estudiantes & $\begin{array}{c}\text { Padres } \\
\text { de } \\
\text { familia }\end{array}$ & Docentes & Directivos \\
\hline Optima & $39 \%$ & $52 \%$ & $41 \%$ & $76 \%$ & $83 \%$ \\
\hline Regular & $52 \%$ & $43 \%$ & $48 \%$ & $24 \%$ & $17 \%$ \\
\hline Insuficiente & $7 \%$ & $5 \%$ & $11 \%$ & $0 \%$ & $0 \%$ \\
\hline Sin conocimiento & $1 \%$ & $0 \%$ & $0 \%$ & $0 \%$ & $0 \%$ \\
\hline total & $\mathbf{1 0 0} \%$ & $\mathbf{1 0 0 \%}$ & $\mathbf{1 0 0 \%}$ & $\mathbf{1 0 0} \%$ & $\mathbf{1 0 0 \%}$ \\
\hline
\end{tabular}

Fuente: La Comunidad Los Bajos Del Pechiche y la Unidad Educativa Dr. Daniel Acosta

Rosales

Realizado por: Estudiantes de Octavo semestre de la Facultad de Trabajo Social, Uleam.

Gráfico 2. ¿Cómo fue la participación de los involucrados en el proyecto de prevención de drogas en los Bajos del Pechiche? 


\section{Gráfico 2}

\section{E Comunidad Estudiantes = Padres de familia m Docentes = Directivos}

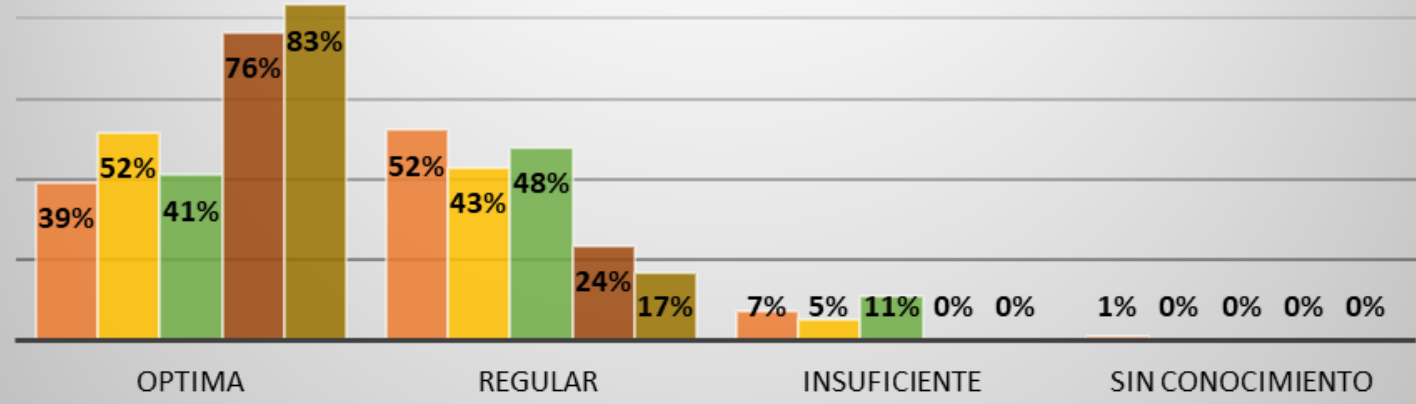

Fuente: La Comunidad Los Bajos Del Pechiche y la Unidad Educativa Dr. Daniel Acosta

Rosales

Realizado por: Estudiantes de Octavo semestre de la Facultad de Trabajo Social, Uleam.

Análisis:

En referencia a los resultados de esta tabla se demuestra que la participación de los involucrados en el proyecto de prevención de drogas fue regular faltando quizás más compromiso por parte de los involucrados del proyecto.

Tabla 3 ¿Cuál es la relevancia de las actividades del proyecto para hacer buen uso del tiempo libre y prevenir el uso de estupefacientes psicotrópicos?

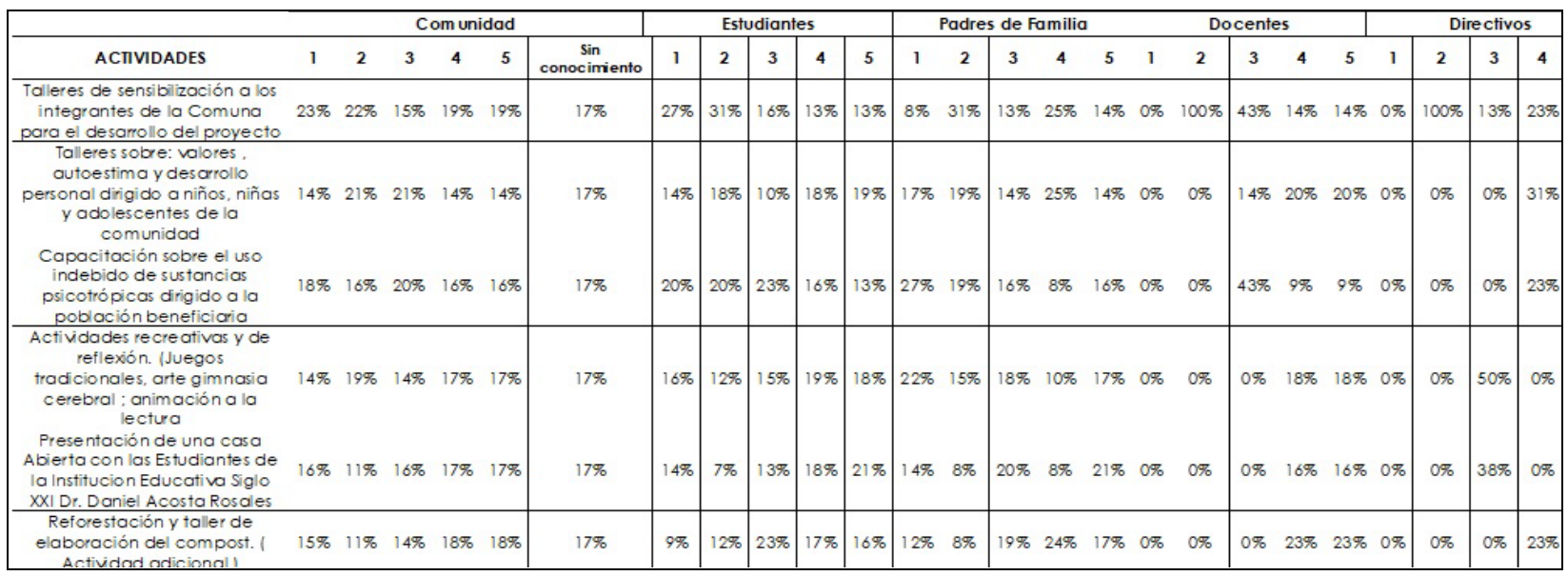


Fuente: La Comunidad Los Bajos Del Pechiche y la Unidad Educativa Dr. Daniel Acosta Rosales

Realizado por: Estudiantes de Octavo semestre de la Facultad de Trabajo Social, Uleam.

Gráfico 3 ¿Cuál es la relevancia de las actividades del proyecto para hacer buen uso del tiempo libre y prevenir el uso de estupefacientes psicotrópicos?

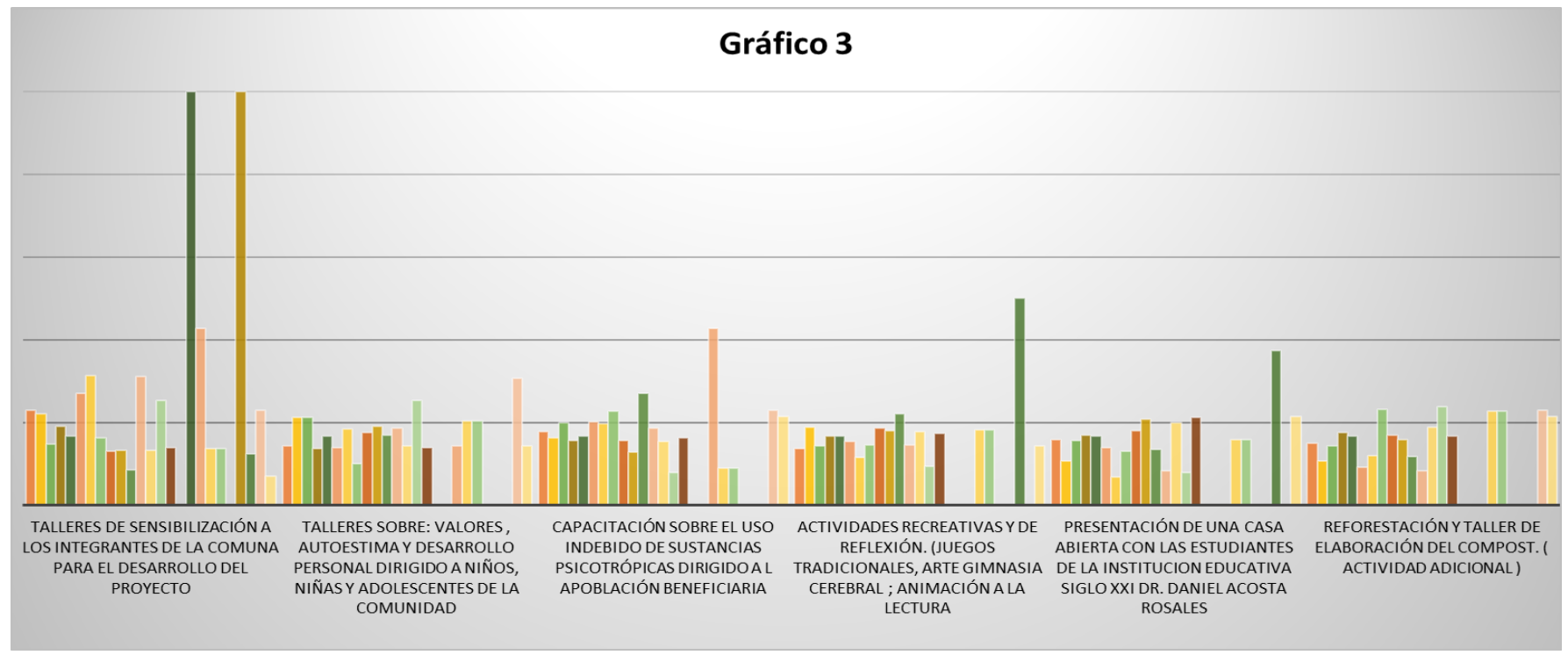

Fuente: La Comunidad Los Bajos Del Pechiche y la Unidad Educativa Dr. Daniel Acosta Rosales

Realizado por: Estudiantes de Octavo semestre de la Facultad de Trabajo Social, Uleam.

\section{Análisis:}

Considerando los resultados obtenidos de la encuesta se demuestra la gran relevancia que tuvieron las actividades del proyecto para hacer buen uso del tiempo libre y prevenir así el consumo de estupefacientes psicotrópicos, de mayor relevancia fue la presentación de una casa abierta con las estudiantes de la Institución Educativa Siglo XXI Dr. Daniel Acosta Rosales. 
Tabla 4 ¿En qué porcentaje considera usted que ha disminuido el consumo de estupefacientes psicotrópicos en los Bajos del Pechiche?

\begin{tabular}{|l|c|c|c|c|c|}
\hline $\begin{array}{l}\text { \% Disminución del } \\
\text { consumo de } \\
\text { estupefacientes en los } \\
\text { Bajos del Pechiche }\end{array}$ & Comunidad & Estudiantes & $\begin{array}{l}\text { Padres } \\
\text { de } \\
\text { familia }\end{array}$ & Docentes & Directivos \\
\hline $0 \%-9 \%$ & $13 \%$ & $11 \%$ & $21 \%$ & $12 \%$ & $17 \%$ \\
\hline $10 \%-25 \%$ & $17 \%$ & $25 \%$ & $15 \%$ & $35 \%$ & $0 \%$ \\
\hline $26 \%-50 \%$ & $29 \%$ & $27 \%$ & $30 \%$ & $24 \%$ & $83 \%$ \\
\hline $51 \%-75 \%$ & $33 \%$ & $28 \%$ & $29 \%$ & $18 \%$ & $0 \%$ \\
\hline $76 \%-100 \%$ & $6 \%$ & $10 \%$ & $5 \%$ & $12 \%$ & $0 \%$ \\
\hline Sin conocimiento & $1 \%$ & $0 \%$ & $0 \%$ & $0 \%$ & $0 \%$ \\
\hline TOTAL & $\mathbf{1 0 0 \%}$ & $\mathbf{1 0 0} \%$ & $\mathbf{1 0 0} \%$ & $\mathbf{1 0 0} \%$ & $\mathbf{1 0 0 \%}$ \\
\hline
\end{tabular}

Fuente: La Comunidad Los Bajos Del Pechiche y la Unidad Educativa Dr. Daniel Acosta Rosales

Realizado por: Estudiantes de Octavo semestre de la Facultad de Trabajo Social, Uleam.

Gráfico 4 ¿En qué porcentaje considera usted que ha disminuido el consumo de estupefacientes psicotrópicos en los Bajos del Pechiche?

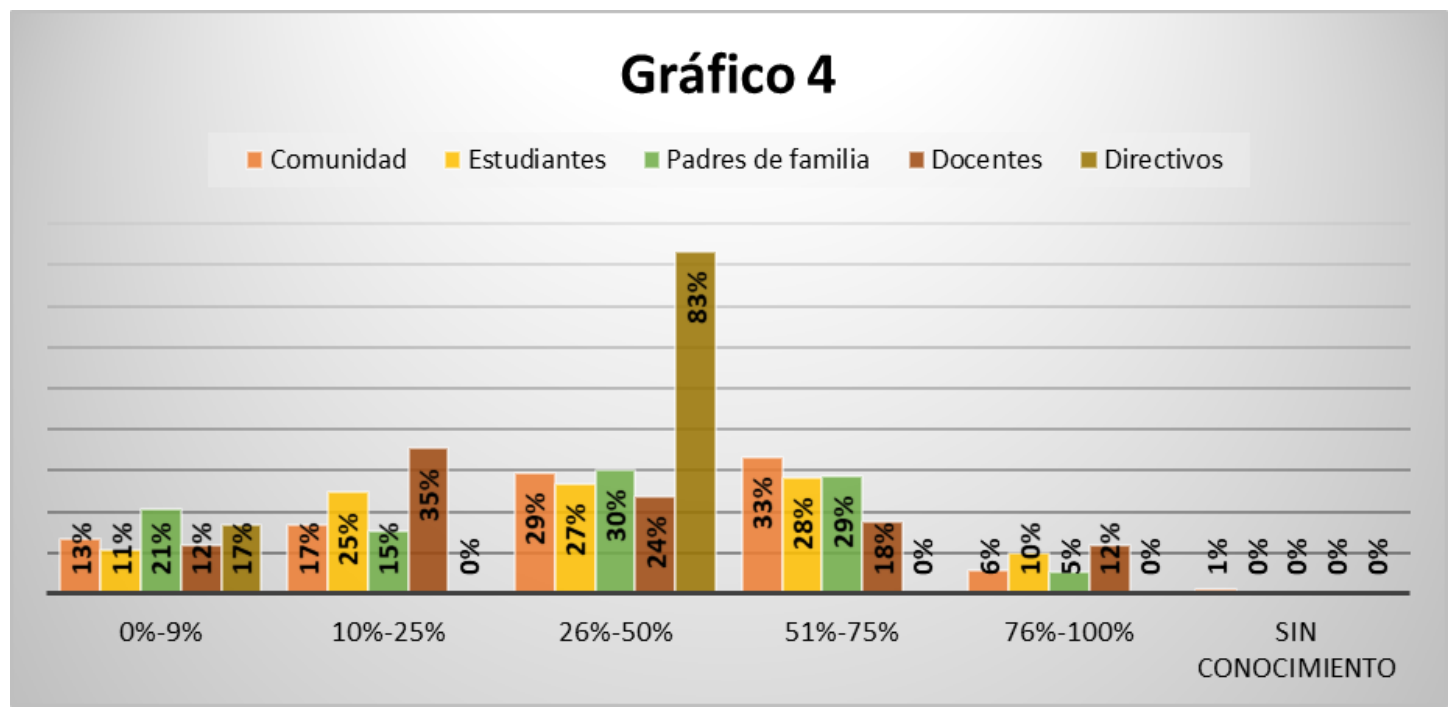

Fuente: La Comunidad Los Bajos Del Pechiche y la Unidad Educativa Dr. Daniel Acosta Rosales

Realizado por: Estudiantes de Octavo semestre de la Facultad de Trabajo Social, Uleam.

\section{Análisis:}

Mediante los resultados obtenidos por las encuestas y evidenciado en esta tabla se demuestra que el consumo de estupefacientes psicotrópicos si ha tenido una disminución dentro del rango 
contemplado del 51\% al 75\% dentro de la Comunidad "Los Bajos Del Pechiche" y la Unidad Educativa Siglo XXI Dr. Daniel Acosta Rosales, evidenciándose un impacto muy importante.

Tabla 5 ¿Considera usted que el proyecto realizado ayudó a optimizar el uso del tiempo libre de los beneficiarios?

\begin{tabular}{|l|c|c|c|c|c|}
\hline $\begin{array}{l}\text { Buen uso del } \\
\text { tiempo libre }\end{array}$ & Comunidad & Estudiantes & $\begin{array}{c}\text { Padres } \\
\text { de } \\
\text { familia }\end{array}$ & Docentes & Directivos \\
\hline Sí & $63 \%$ & $70 \%$ & $52 \%$ & $82 \%$ & $67 \%$ \\
\hline No & $11 \%$ & $10 \%$ & $33 \%$ & $12 \%$ & $0 \%$ \\
\hline $\begin{array}{l}\text { Solo en el momento } \\
\text { en que se llevaron a } \\
\text { efecto las } \\
\text { actividades }\end{array}$ & $26 \%$ & $20 \%$ & $15 \%$ & $6 \%$ & $33 \%$ \\
\hline Sin conocimiento & $1 \%$ & $0 \%$ & $0 \%$ & $0 \%$ & $0 \%$ \\
\hline TOTAL & $\mathbf{1 0 0 \%}$ & $\mathbf{1 0 0 \%}$ & $\mathbf{1 0 0 \%}$ & $\mathbf{1 0 0 \%}$ & $100 \%$ \\
\hline
\end{tabular}

Fuente: La Comunidad Los Bajos Del Pechiche y la Unidad Educativa Dr. Daniel Acosta Rosales

Realizado por: Estudiantes de Octavo semestre de la Facultad de Trabajo Social, Uleam.

Gráfico 5 ¿Considera usted que el proyecto realizado ayudó a optimizar el uso del tiempo libre de los beneficiarios?

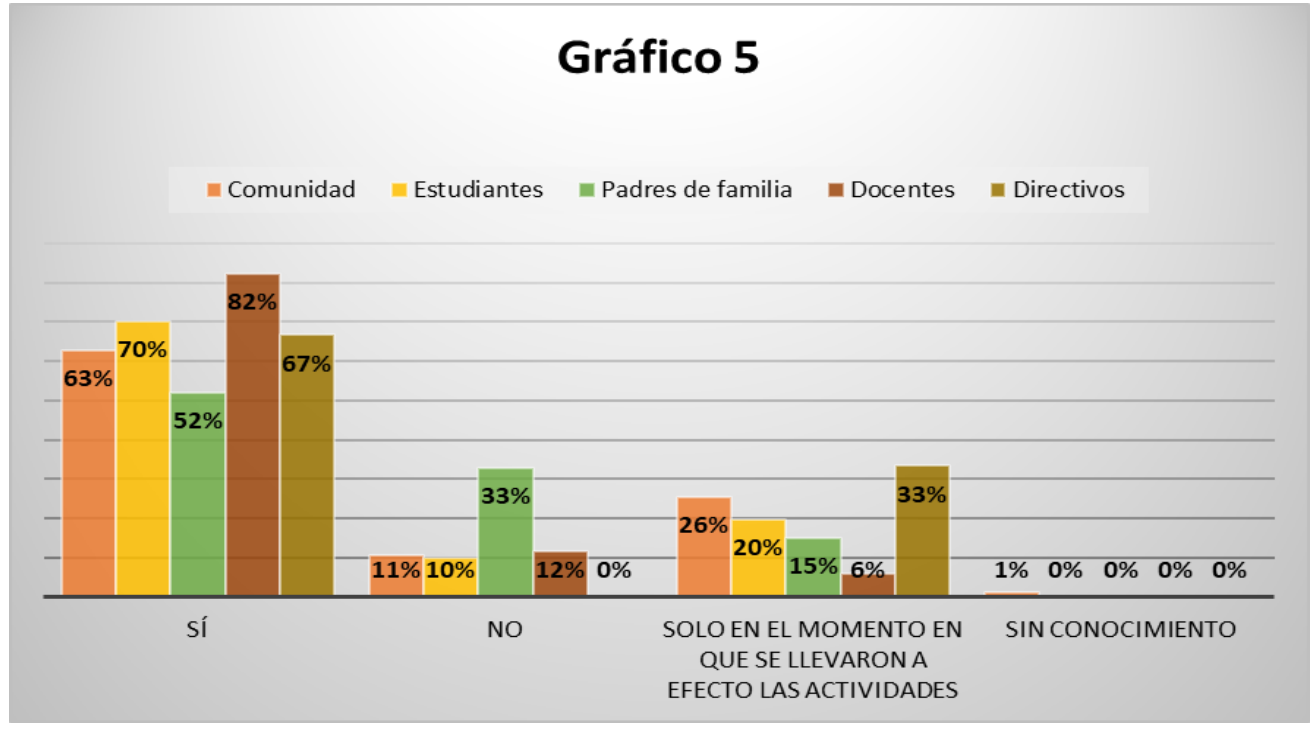

Fuente: La Comunidad Los Bajos Del Pechiche y la Unidad Educativa Dr. Daniel Acosta Rosales Realizado por: Estudiantes de Octavo semestre de la Facultad de Trabajo Social, Uleam.

\section{Análisis:}


Según los resultados se demuestra que las actividades del proyecto ayudaron a optimizar el buen uso del tiempo libre y no dejarse tentar por el consumo de sustancias psicotrópicas, lo que beneficia a los estudiantes de la Unidad Educativa Siglo XXI Dr. Daniel Acosta Rosales, mejorando su calidad devida y por ende a la comunidad toda.

Tabla 6 ¿Considera usted que el proyecto abarcó a todos los beneficiarios?

\begin{tabular}{|l|c|c|c|c|c|}
\hline $\begin{array}{l}\text { Buen uso del } \\
\text { tiempo libre }\end{array}$ & Comunidad & Estudiantes & $\begin{array}{c}\text { Padres } \\
\text { de } \\
\text { familia }\end{array}$ & Docentes & Directivos \\
\hline 500 & $38 \%$ & $42 \%$ & $55 \%$ & $59 \%$ & $67 \%$ \\
\hline 750 & $28 \%$ & $44 \%$ & $22 \%$ & $18 \%$ & $33 \%$ \\
\hline 350 & $33 \%$ & $14 \%$ & $23 \%$ & $24 \%$ & $0 \%$ \\
\hline Sin conocimiento & $1 \%$ & $0 \%$ & $0 \%$ & $0 \%$ & $0 \%$ \\
\hline TOTAL & $\mathbf{1 0 0 \%}$ & $\mathbf{1 0 0 \%}$ & $\mathbf{1 0 0 \%}$ & $\mathbf{1 0 0 \%}$ & $\mathbf{1 0 0 \%}$ \\
\hline
\end{tabular}

Fuente: La Comunidad Los Bajos Del Pechiche y la Unidad Educativa Dr. Daniel Acosta Rosales

Realizado por: Estudiantes de Octavo semestre de la Facultad de Trabajo Social, Uleam.

Gráfico 6 ¿Considera usted que el proyecto abarcó a todos los beneficiarios?

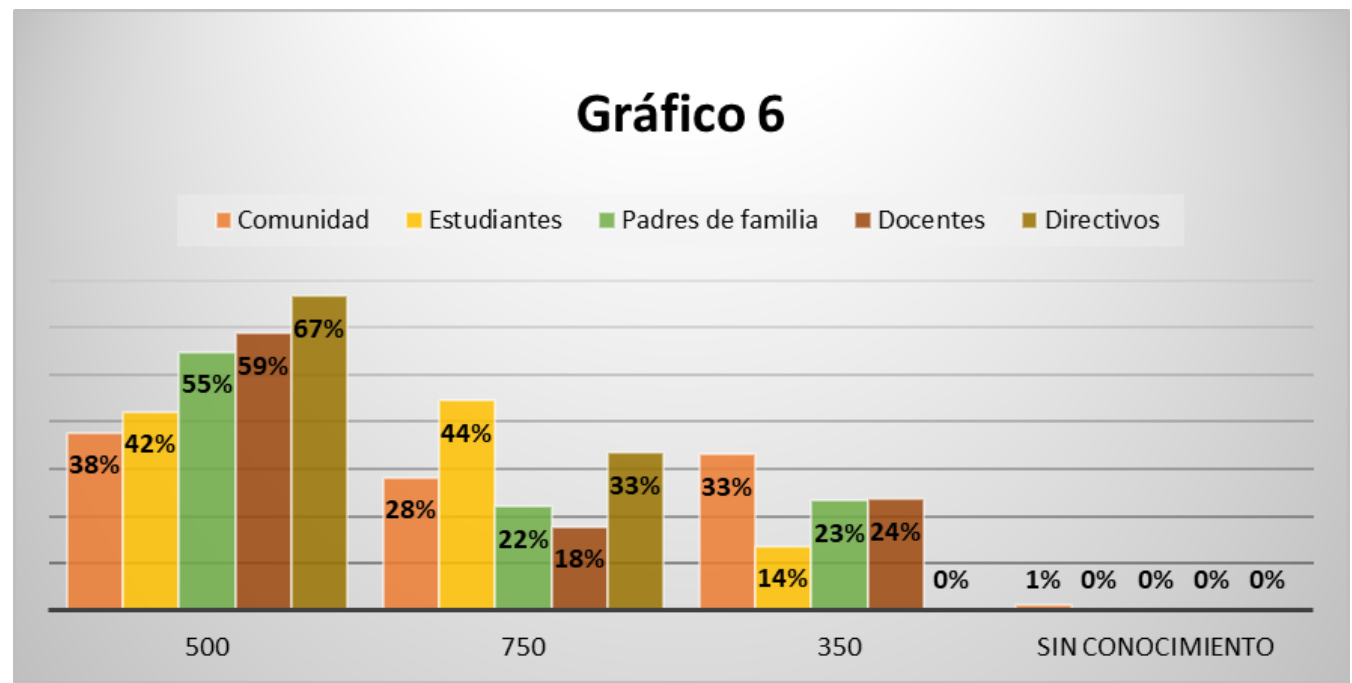

Fuente: La Comunidad Los Bajos Del Pechiche y la Unidad Educativa Dr. Daniel Acosta Rosales

Realizado por: Estudiantes de Octavo semestre de la Facultad de Trabajo Social, Uleam. 


\section{Análisis:}

En referencia a los resultados de esta tabla podemos observar que se pudo abarcar a un número considerable de beneficiarios, entre ellos estudiantes, docentes, padres de familia y comunidad en general, lo que implica que el mensaje de prevención fue difundido en toda la comunidad.

Tabla 7 ¿Considera que este proyecto generó un impacto positivo en los involucrados?

\begin{tabular}{|l|c|c|c|c|c|}
\hline $\begin{array}{l}\text { IMPACTO } \\
\text { POSITIVO }\end{array}$ & Comunidad & Estudiantes & $\begin{array}{c}\text { Padres de } \\
\text { familia }\end{array}$ & Docentes & Directivos \\
\hline SI & $64 \%$ & $59 \%$ & $38 \%$ & $94 \%$ & $100 \%$ \\
\hline NO & $9 \%$ & $6 \%$ & $40 \%$ & $6 \%$ & $0 \%$ \\
\hline TAL VEZ & $26 \%$ & $35 \%$ & $22 \%$ & $0 \%$ & $0 \%$ \\
\hline $\begin{array}{l}\text { SIN } \\
\text { CONOCIMIENTO }\end{array}$ & $1 \%$ & $0 \%$ & $0 \%$ & $0 \%$ & $0 \%$ \\
\hline TOTAL & $\mathbf{1 0 0} \%$ & $\mathbf{1 0 0} \%$ & $\mathbf{1 0 0} \%$ & $\mathbf{1 0 0} \%$ & $100 \%$ \\
\hline
\end{tabular}

Fuente: La Comunidad Los Bajos Del Pechiche y la Unidad Educativa Dr. Daniel Acosta Rosales

Realizado por: Estudiantes de Octavo semestre de la Facultad de Trabajo Social, Uleam.

Gráfico 7 ¿Considera que este proyecto generó un impacto positivo en los involucrados?

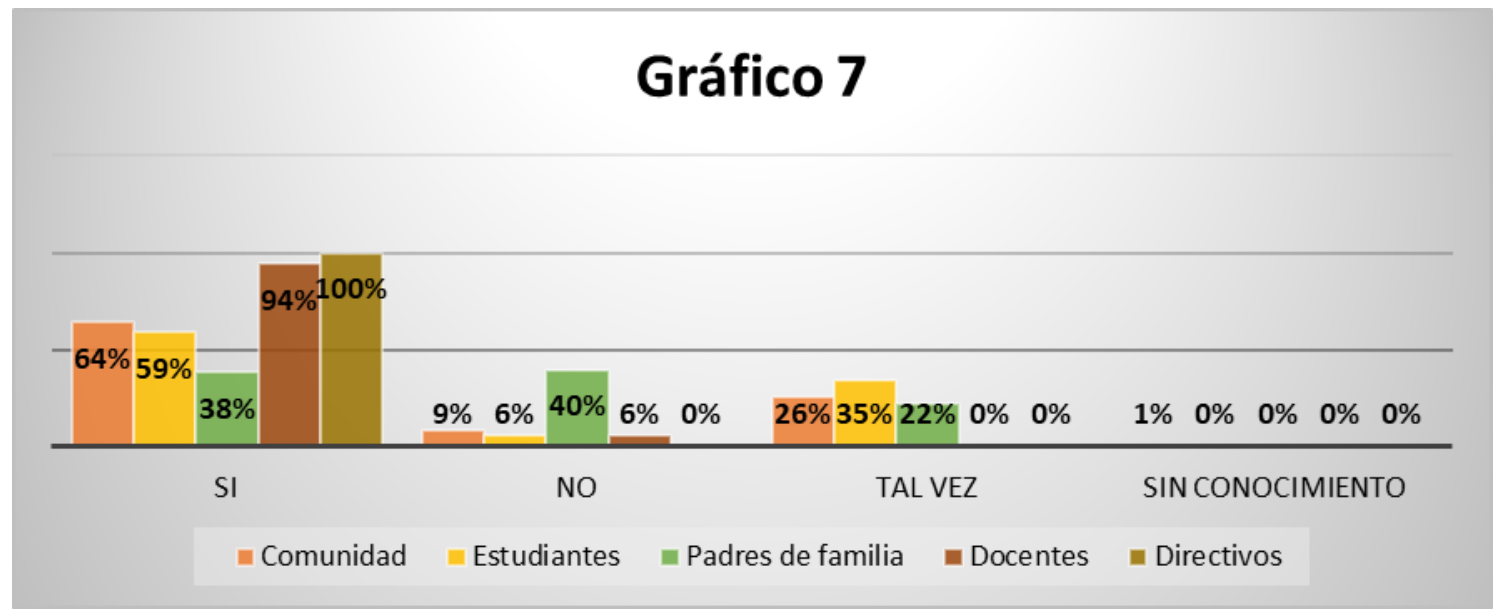

Fuente: La Comunidad Los Bajos Del Pechiche y la Unidad Educativa Dr. Daniel Acosta Rosales

Realizado por: Estudiantes de Octavo semestre de la Facultad de Trabajo Social, Uleam.

\section{Análisis:}

De acuerdo a la información obtenida por la encuesta realizada a La Comunidad "Los Bajos Del Pechiche" y la Unidad Educativa Siglo XXI Dr. Daniel Acosta Rosales indican los resultados de la tabla que el desarrollo del proyecto ha causado un impacto positivo en los involucrados. 


\section{Conclusión}

El flagelo de la drogadicción es un mal social que incide en la conducta de quienes incurren en tal enfermedad, lo cual puede traducirse a la larga en problemas sociales de conducta e incluso de participación en conductas disruptivas e incluso delictivas. Los niños y los adolescentes son individuos en proceso de formación de su personalidad y por ende requieren el soporte psicológico y social que le puede brindar la familia, la educación y los agentes sociales educativos, tales como los medios de comunicación, las amistades y otras instituciones como organizaciones de filiación deportiva, cultural, artística, religiosa o política.

Por consiguiente, la familia, la escuela, la comunidad y los maestros poseen un rol fundamental en el proceso de prevención contra la drogadicción en este grupo etario de niños y jóvenes de entre 12 y 15 años. Es así como se hace conciencia de la relevancia de abordar este tema, ejecutar programas de prevención y uso adecuado del tiempo libre de los niños y jóvenes de esta comunidad, así como las opiniones y caracterización de los maestros que atienden los estudiantes de la referida institución educativa en cuestión.

De los resultados obtenidos en la aplicación de encuesta y criterios dados en las entrevistas a docentes, directivos, padres de familia, estudiantes y comunidad, se afirma que se logró el objetivo de describir las opiniones y conocimientos de estos niños y docentes con respecto a este tema, de cuyos resultados se deriva que la ejecución de las charlas de prevención y actividades de uso adecuado de tiempo libre, generaron un impacto muy positivo en los niños y jóvenes especialmente, creándose un espacio de reflexión y desarrollo de criterios de conciencia frente a este gran mal que azota a la humanidad. 


\section{Referencias Bibliográficas}

Arias, F., 2006. El proyecto de investigación. Introducción a la metodología científica. $5^{\text {a }}$ Edición. Editorial Epistame, C.A. Caracas - Venezuela.

Branden, N., 2001.La Psicología de la autoestima Editorial: Paidos Iberica. ISBN: 9788449327001

García, v (2000). Familia, Sexo y Droga. Ediciones RIALP, Madrid. España. ISBN: 84-321$1900-8$

Hernández, S., Fernández, C., y Baptista, L., 2006. Metodología de la investigación. (5ª ed.). México: McGraw-Hill, Interamericana de México, S.A.

Lewis, H., 1999. La cuestión de los valores humanos. Editorial: GEDISA. ISBN: 8474325145 ISBN-13: 9788474325140.

Marcos, R., 2007. La autoestima. Editorial: S.L.U. ESPASA LIBROS ISBN: 9788467036992.

Robín S. Sharma, 2010. El líder que no tenía cargo. Editorial: Grijalbo, Barcelona. ISBN 10:8425344425 ISBN -13: 9788425344428. 
